\title{
Combination of Asymmetric PCMA and Common Multiple Access Technology
}

\author{
Xingchen $\mathrm{Xu}^{\mathrm{a}}$, Jian Cheng ${ }^{\mathrm{b}}$, Jingyu Tang ${ }^{\mathrm{c}}$, Chuang Wang ${ }^{\mathrm{d}}$, Xiaoyu Cui ${ }^{\mathrm{e}}$ and \\ Xuefei Liang ${ }^{f}$
}

Army Engineering University of PLA, Nanjing 210000, China.

atammyxxc@163.com, bchjatice@163.com, 'cmrtangjingyu@qq.com, dchuangw91@163.com, exiaoyu940528@sina.cn, f18362973763@163.com

\begin{abstract}
Aiming at the transmission efficiency of asymmetric PCMA communication and the improvement of its communication capacity, the full use of the characteristics that PCMA technology can be combined with common multiple access technology can be realized by transmitting multiple small signals in a large signal band. Firstly, the method of asymmetric PCMA combined with FDMA and CDMA is introduced in principle. Then, the influence of parameter estimation error on the demodulation performance of asymmetric PCMA under different multiple access technologies is analyzed by simulation. Finally, for two kinds of multiple access technologies, Corresponding analysis and conclusions are given after comparison.
\end{abstract}

Keywords: Asymmetric, Paired Carry Multiple Access (PCMA), Multiple Access (MA), Performance analysis.

\section{Introduction}

PCMA communication technology is a new type of satellite multiple access technology, which is suitable for two-way satellite communication systems using transparent transponders, and can be used with Frequency Division Multiple Access (FDMA) or Code Division Multiple Access (CDMA). Multiple access technology is used in combination[1]. Since the PCMA technology allows the communication parties to simultaneously communicate through the satellite transponder using the same frequency and CDMA code, this way of superimposing the signal in the time-frequency domain effectively saves spectrum resources, improves the utilization of the frequency band, and this also doubles the communication capacity.

In order to further improve the transmission efficiency of asymmetric PCMA communication and its communication capacity, a form of transmitting multiple small signals in a large signal band can be adopted. It is known that the PCMA communication technology can be used in combination with a commonly used multiple access technology for convenience. Description, this chapter refers to the combination of asymmetric PCMA and frequency division multiple access technology as APCMA-FDMA (Asymmetric Paired Carrier Multiple Access-Frequency Division Multiple Access, APCMA-FDMA), and the code division multiple access technology the combination is called APCMA-CDMA (Asymmetric Paired Carrier Multiple Access-Code Division Multiple Access, APCMA-CDMA). In this chapter, the principle of combining asymmetric PCMA signals with different multiple access technologies is expounded. Then, the influence of parameter estimation error on the demodulation performance of asymmetric PCMA signals under different multiple access technologies is analyzed by simulation. Finally, the comparison is made to make a corresponding conclusion.

\section{Principles of APCMA-FDMA and APCMA-CDMA}

Frequency Division Multiple Access (FDMA) and Code Division Multiple Access (CDMA) are two common multiple access technologies that are widely used in various communication systems. In this paper, the asymmetric PCMA communication system is adopted to improve the transmission efficiency of the system and expand the communication channel capacity by combining with frequency division multiple access or code division multiple access. This section mainly introduces its principle. 


\subsection{APCMA-FDMA}

Frequency Division Multiple Access (FDMA) is the first MA (Multiple Access, MA) technology implemented on satellite systems. Its principle and operation are simple. Figure 4-1 connects three frequency stations to a frequency transform (Frequency Transform, FT) satellite transponder, a transparent transponder, is used as an example to give a functional diagram of the FDMA process. A specific frequency band $\left(f_{1}, f_{2}, f_{3}\right)$ is assigned to the uplink of each ground station. The frequency/time diagram in the figure shows that each ground station monopolizes its own frequency band or frequency slot, which can be pre-allocated or changed as needed. A guard band is usually added between user bands to avoid mutual interference. The size of the guard band is related to the accuracy and stability of the carrier frequency of the transmitting and receiving ground station, and also to the difference of the maximum Doppler shift between adjacent signals. Therefore, the guard band set in the FDMA should be larger than any carrier signal. The maximum drift value relative to its nominal frequency[2].

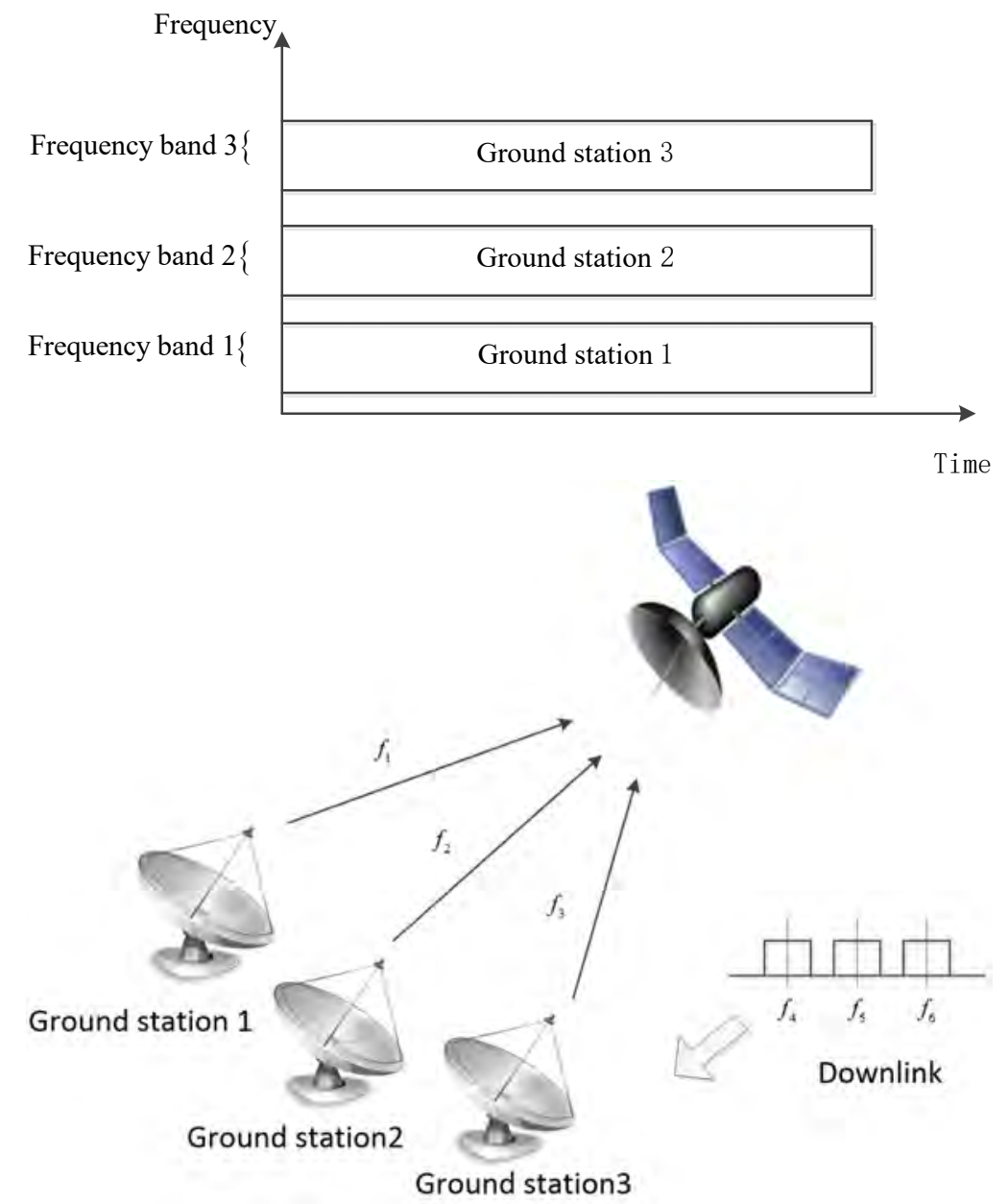

Fig. 1 FDMA

When the signal goes down, because the carrier spectrum passes through the frequency-converting satellite, the ground station needs to tune the receiver to a specific downlink frequency to receive the transmitting carrier of the corresponding uplink ground station. And because the entire FDMA spectrum is transmitted on the downlink, that is, multiple carriers exist at the same time, the ground receiving station must be able to receive the entire spectrum and filter it to distinguish the carrier actually sent to the station, and send it to other grounds. Carrier filtering of the station.

According to whether each ground station uses multiplexing technology in the transmission carrier, FDMA is divided into two categories: FDMA (Multiple Channels Per Carrier-Frequency Division 
Multiple Access, MCPC-FDMA) and single channel per carrier. FDMA (Single Channel Per CarrierFrequency Division Multiple Access, SCPC-FDMA).

The asymmetric PCMA signal is a mixed signal obtained by superimposing a wide-band, highpower primary station signal and a narrow-band, low-power small station signal, so that the frequency band of the primary station signal can be divided into multiple frequency bands for use by the small station signal. And the small signal code rate is smaller than the large signal code rate, and each small signal is distinguished by different carrier frequencies, and its spectrum structure is shown in Figure 2. When the primary station receives the APCMA-FDMA signal, in order to realize the demodulation of the small station signal, it is necessary to reconstruct the self-interference signal, that is, the primary station signal. The spectrum diagram is shown in Figure 3, and then the bandpass is passed. The filter filters out the corresponding small signal, and finally selects the desired carrier to demodulate the small signal.

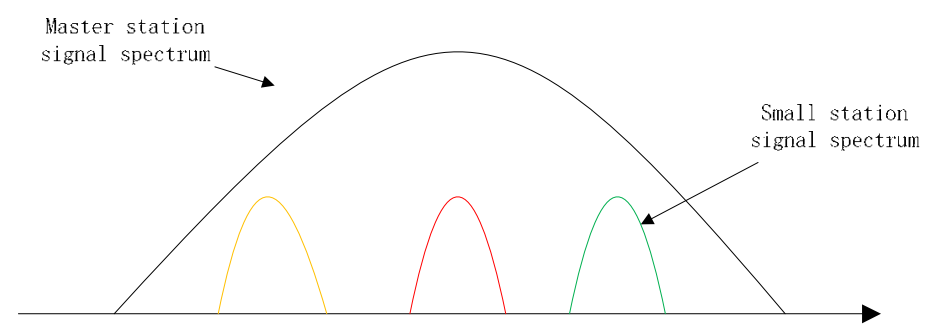

Fig. 2 APCMA-FDMA signal spectrum

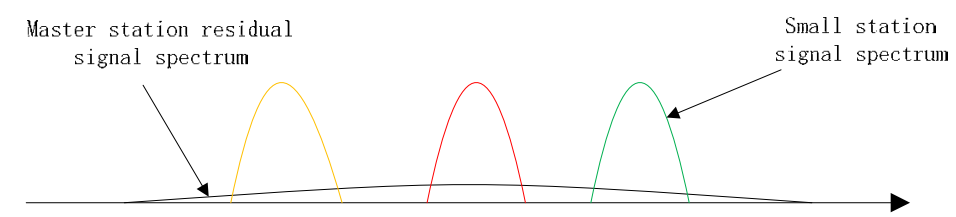

Fig. 3 Reconstruction canceled APCMA-FDMA signal spectrum

\subsection{APCMA-CDMA}

Code Division Multiple Access (CDMA) is a basic multiple access technology, which implements signal segmentation according to the orthogonality of address codes. The basic principle is to use a periodic code sequence with very strong autocorrelation properties and weak crosscorrelation properties as an address. The information is the address code, and the carrier modulated by the user information is remodulated to greatly expand the spectrum, which is also called spread spectrum modulation. After the satellite channel transmission, the receiving end uses the locally generated known address code as a reference, and discriminates all the received signals according to the difference of correlation, and restores the wideband signal whose address code is completely consistent with the local address code to a narrowband signal. When selected, other signals unrelated to the local address code are kept or expanded into bandwidth signals to be filtered out. This method is also called correlation detection or spread spectrum modulation[3]. Figure 4 shows the functional block diagram of the CDMA process. To transmit ground station data packets, each uplink earth station is assigned a time slot and frequency band in the coding sequence. The downlink transmits a cross sequence of all packets, as shown in Figure 4-3. The downlink receiving station must know the encoding of the frequency and time position in order to detect the complete data sequence. The receiving ground station that knows the encoding can recover the signal from a signal that appears to be noisy to the receiver that does not know the encoding. Since the CDMA process has the characteristics of signal spreading, CDMA is often called Spread Spectrum or Spread Spectrum Multiple Access (SSMA)[4]. 

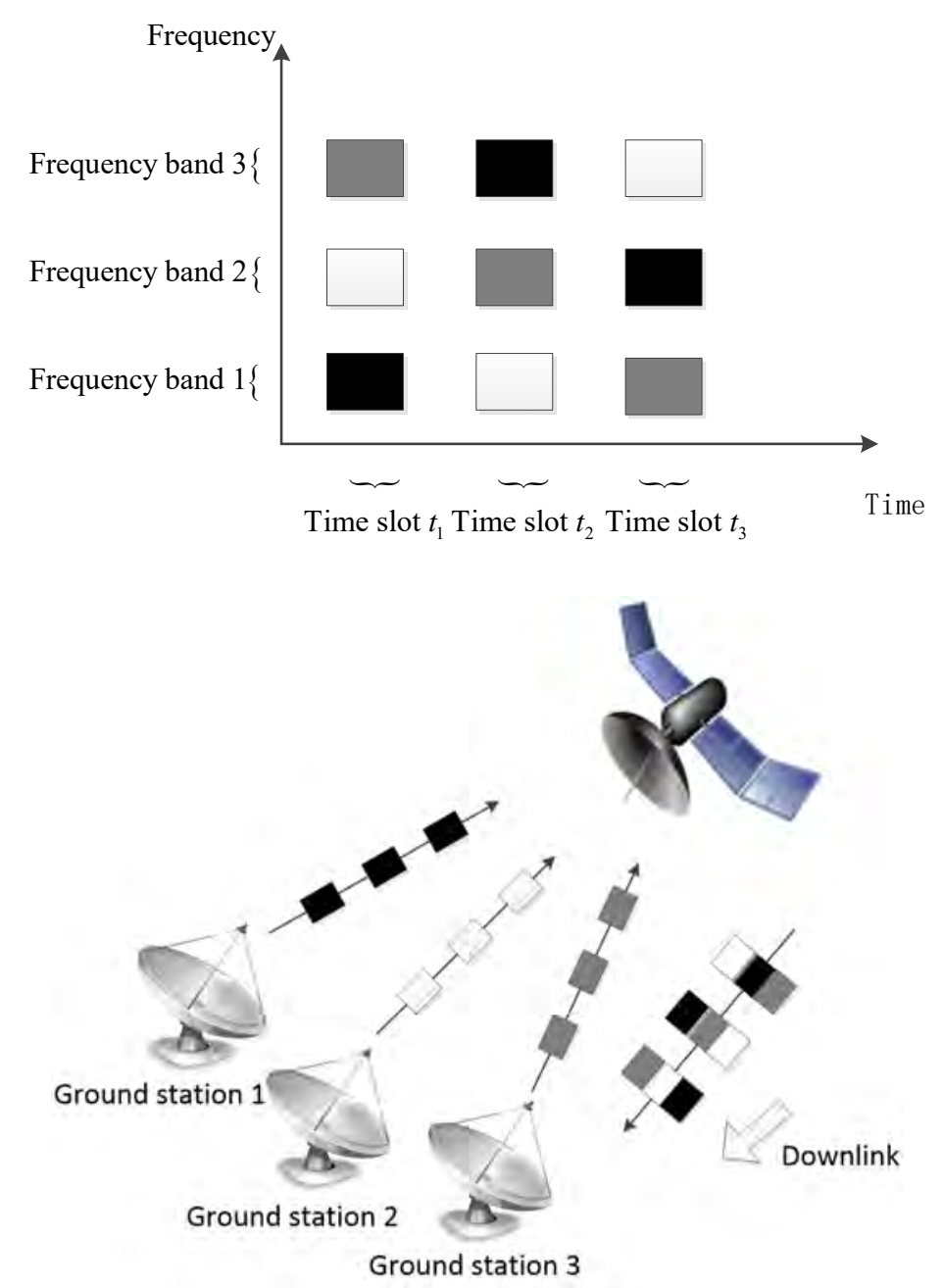

Fig. 4 CDMA

In the asymmetric PCMA application, the bandwidth of the small station signal is set to be substantially the same as the bandwidth of the primary station signal, and the small station signal code rate is smaller than the primary station signal code rate, and each small station signal is distinguished by different spreading code words. Its spectrum structure is shown in Figure 5. When the primary station receives the APCMA-CDMA signal, in order to demodulate the small station signal, it is necessary to reconstruct the self-interference signal, that is, the primary station signal. The spectrum diagram is shown in Figure 6, and then despread. The small station signal is multiplied by its corresponding spreading code word, and finally demodulated by the CDMA demodulation method, and the other small signal and noise interference can be filtered through the low-pass filter.

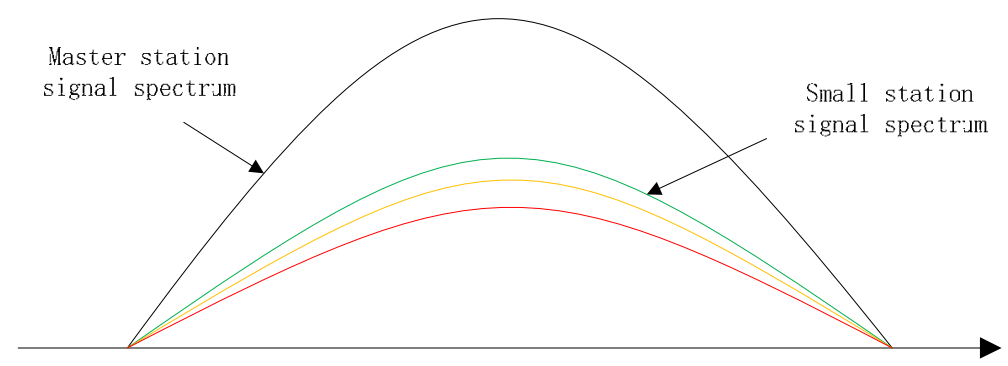

Fig. 5 APCMA-CDMA signal spectrogram 


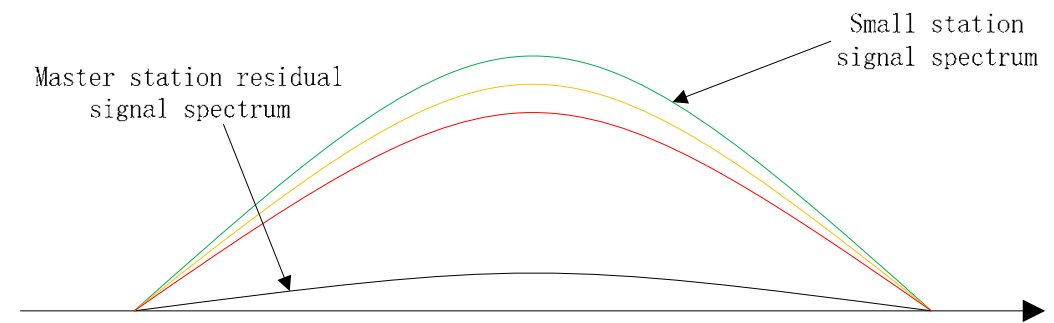

Fig. 6 Reconstruction canceled APCMA-CDMA signal spectrogram

\section{APCMA-FDMA and APCMA-CDMA Performance Simulation}

In the previous section, the principle of combining FDMA and CDMA multiple access technology with PCMA technology is described. The main purpose is to improve the transmission efficiency and communication capacity of the communication system by multiplexing the small station signals in the asymmetric PCMA. The coexistence of multiple small station signals is realized by two forms of APCMA-FDMA and APCMA-CDMA. This section focuses on the effects of parameter estimation errors on the demodulation performance of asymmetric PCMA signals in two modes.

\subsection{APCMA-FDMA Simulation}

The basic principle of APCMA-FDMA is given in 2.1. In this section, the Matlab simulation experiment is carried out, and the demodulation performance under different parameter estimation errors is simulated. The parameters are set as follows: the main station signal and the small station signal modulation mode are both QPSK, the signal amplitude ratio is $\frac{h_{1}}{h_{2}}=4$, wherein the main station signal information rate is $200 \mathrm{Kbps}$, the small station signal information rate is $2 \mathrm{Kbps}$, a total of 4 small station signals, the main station signal carrier The frequency is $1 \mathrm{MHz}$, and the carrier frequency of the small station signal is $0.925 \mathrm{MHz}, 0.975 \mathrm{MHz}, 1.025 \mathrm{MHz}, 1.075 \mathrm{MHz}$, and the sampling frequency is $8 \mathrm{MHz}$. At the same time, the standard deviation of the normal phase error is set to $\sigma_{p}=\frac{\pi}{90}, \frac{\pi}{45}$, and the mean value is zero; the relative values of the amplitude errors $R_{h}\left(R_{h}=\frac{\Delta h}{h_{1}}\right)$ is $5 \%$ and $10 \%$, respectively.

Figure 7 shows the bit error rate curves of APCMA-FDMA under different normal phase error conditions. Figure 8 shows the bit error rate curves of APCMA-FDMA under different amplitude error conditions. By comparison, the normal phase error and the amplitude estimation error have little effect on the APCMA-FDMA signal. The reason is that when the bandpass filter is used to extract the signals of each small station, the filter will filter out most of the main station signals. The signal residual caused by the offset error, the interference of the residual part of the primary station signal to the small station signal is reduced, so the normal phase error and the amplitude estimation error have little influence on the bit error rate of the APCMA-FDMA signal. 


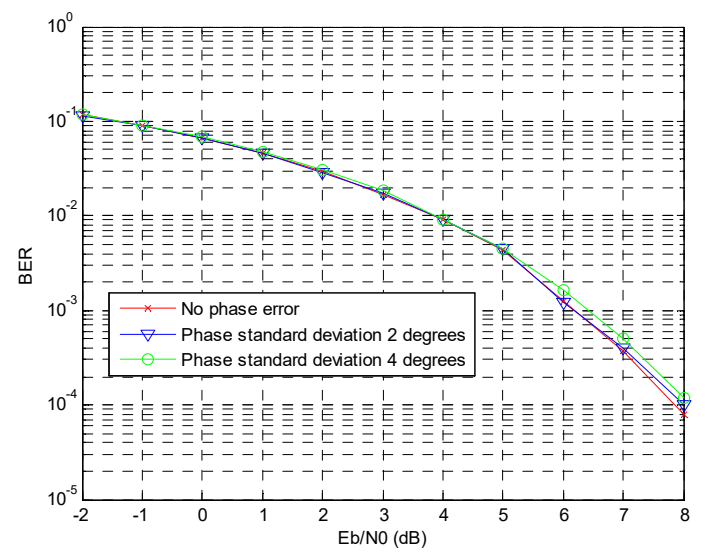

Fig. 7 Normal phase error affects APCMA-FDMA

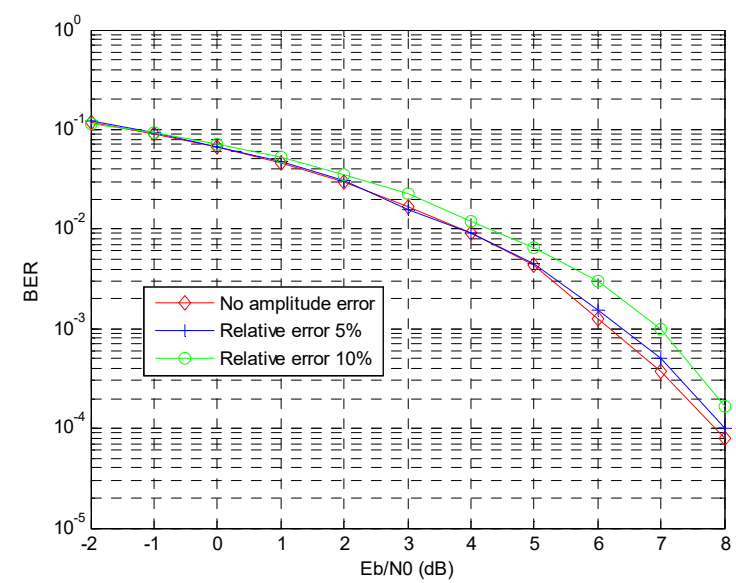

Fig. 8 Amplitude error affects APCMA-FDMA

\subsection{APCMA-CDMA Simulation}

The basic principle of the combination of asymmetric PCMA and CDMA multiple access technology is described in the previous section 2.2. This section performs Matlab simulation experiments to simulate the demodulation performance of APCMA-CDMA signals under different parameter estimation errors. The parameter settings are as follows: the primary station signal and the small station signal modulation mode are both QPSK, the signal amplitude ratio is $\frac{h_{1}}{h_{2}}=4$, the information rate of the primary station signal is $200 \mathrm{Kbps}$, the information rate of the small station signal is $2 \mathrm{Kbps}$, a total of 4 small station signals, the spreading code The word uses a chaotic sequence with a code length of 100 . The carrier frequency of the large and small signals is $1 \mathrm{MHz}$, and the sampling frequency is $8 \mathrm{MHz}$. At the same time, the standard deviation of the normal phase error is set to $\sigma_{p}=\frac{\pi}{90}, \frac{\pi}{45}$, and the mean value is zero; the relative value of the amplitude error $R_{h}\left(R_{h}=\frac{\Delta h}{h_{1}}\right)$ of the amplitude error is $5 \%$ and $10 \%$, respectively.

Figure 9 shows the bit error rate curves of APCMA-CDMA under different normal phase error conditions. Figure 10 shows the bit error rate curves of APCMA-CDMA under different amplitude error conditions. By comparison, the normal phase error and amplitude estimation error have little effect on the APCMA-CDMA signal. The reason is similar to APCMA-FDMA. When extracting each APCMA-CDMA signal, the filter filters out most of the main station signals. Due to the signal residual caused by the cancellation error, the interference of the residual part on the small station signal is reduced, so that the parameter estimation error has a reduced influence on the demodulation performance. 


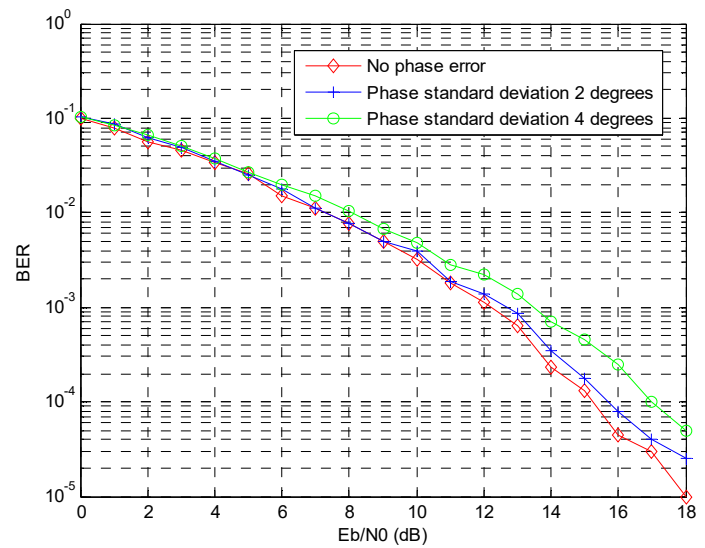

Fig. 9 Normal phase error affects APCMA-CDMA

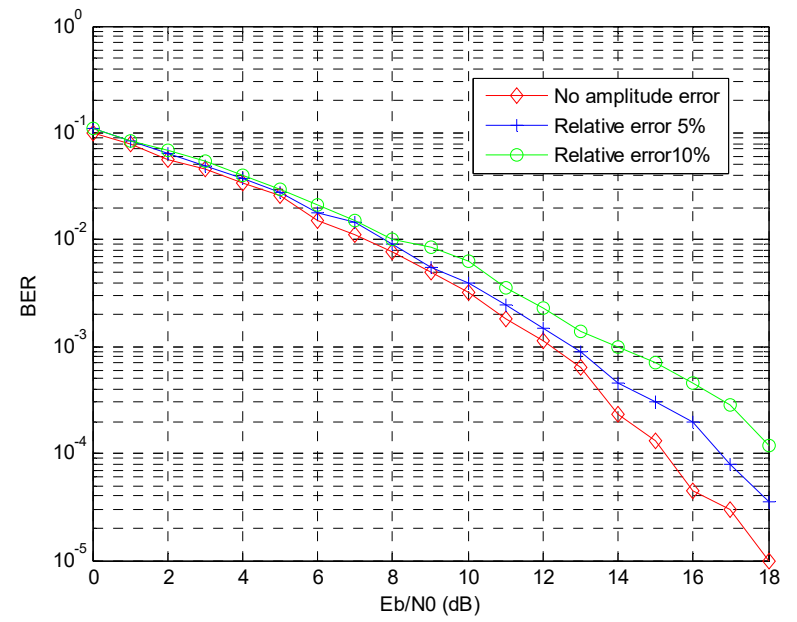

Fig. 10 Amplitude error affects APCMA-CDMA

\section{APCMA-FDMA and APCMA-CDMA Performance Comparison}

By comparing Fig. 7 and Fig. 8, Fig. 9 and Fig. 10, it can be seen that the APCMA-FDMA signal has better demodulation performance than the APCMA-CDMA signal in the channel environment with only Gaussian white noise. Demodulation performance. However, due to the inherent characteristics of direct sequence spread spectrum communication, the demodulation performance of the APCMA-CDMA signal is better than that of the APCMA-FDMA signal in the presence of narrowband interference or Doppler effect. Moreover, from the perspective of information security, the APCMA-CDMA signal can be used for covert communication, and under the cover of large signals, each directly spread small signal is concealed. The reason is shown in the figure. Figures 49 and 4-10 are spectrum diagrams of the APCMA-FDMA signal and the APCMA-CDMA signal, respectively. It can be seen from the figure that there is a distinct spectral peak of the small signal in the spectrum of the APCMA-FDMA signal, which makes the small signal easy to be found. In the spectrum of the APCMA-CDMA signal, the spectrum of the large signal buryes the spectrum of the small signal, and its signal the difference between the spectrum and the simple large signal spectrum is small, which is conducive to the hiding of small signals, thereby improving the security of information transmission. 


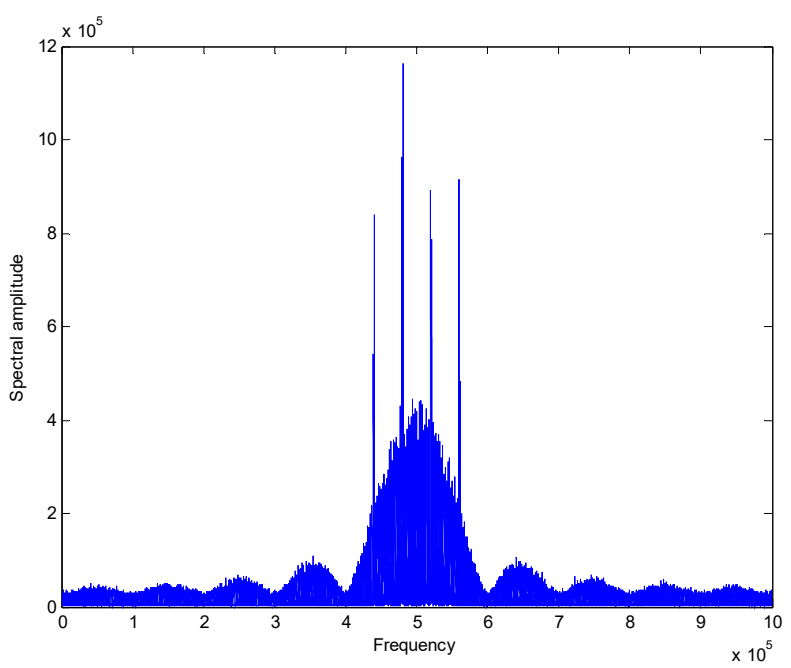

Fig. 11 APCMA-FDMA signal spectrum

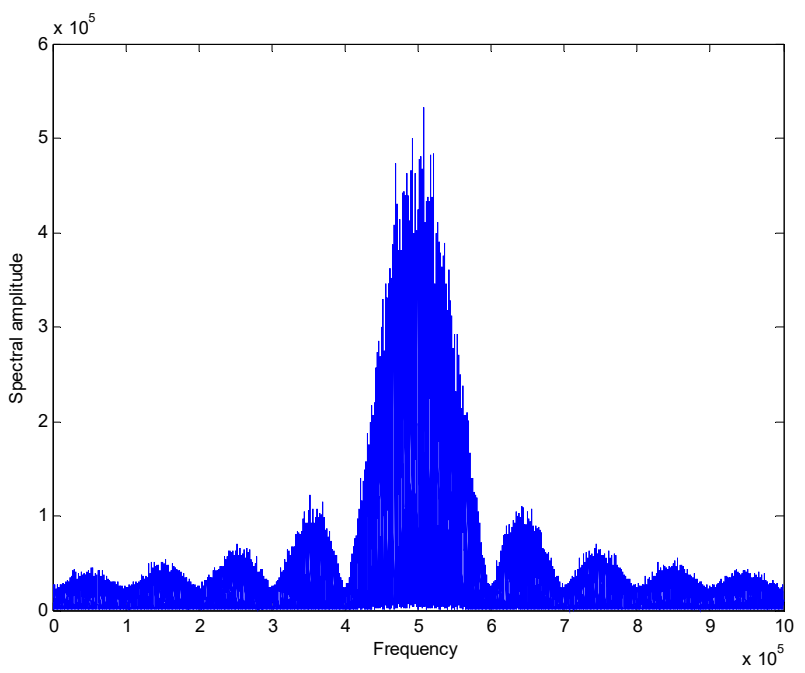

Fig. 12 APCMA-CDMA signal spectrogram

\section{Summary}

In order to improve the transmission efficiency and communication capacity of the asymmetric PCMA communication system, this chapter makes full use of the characteristics that PCMA technology can be combined with the commonly used multiple access technology to transmit multiple small signals in the large signal band to achieve the goal. In principle, the principle of asymmetric PCMA combined with FDMA and CDMA is introduced in principle in Section 2. Section 3 Simulation Analysis The effect of parameter estimation error on the demodulation performance of asymmetric PCMA under different multiple access technologies. Section 4 compares the advantages and disadvantages of combining two kinds of multiple access technologies with PCMA and concludes by comparison.

\section{References}

[1]. Gong Bo. Research on the Paired Carrier Multiple Access technique in satellite communication system [D]. Harbin Institute of Technology, 2010.

[2]. Ippolito L J. Satellite Communications Systems Engineering: Atmospheric Effects, Satellite Link Design and System Performance (Wireless Communications and Mobile Computing) [M]. 2008. 
[3]. Hu Yi. Statistical Study on the Capturing Performance of PN Codes in Spread Spectrum Communication[J]. System Engineering and Electronics, 1992(08):13-20.

[4]. Ippolito. Satellite Communication System Engineering [M]. National Defense Industry Press, 2012. 